\title{
$\mathrm{Au}$ 纳米粒子表面等离子共振效应(SPR)增强 $\mathrm{Au} / \mathrm{Bi}_{2} \mathrm{WO}_{6}$ 异质纳米结构的光催化活性
}

\author{
王丹军, 王婵, 赵强, 郭莉, 杨晓, 吴娇, 付 峰 \\ (延安大学 化学与化工学院, 陕西省化学反应工程重点实验室, 延安 716000)
}

摘 要: 采用水热-光还原法在三维 $\mathrm{Bi}_{2} \mathrm{WO}_{6}$ 的二级结构纳米片表面原位沉积 $\mathrm{Au}$ 纳米粒子 $(\mathrm{Au} \mathrm{NPs})$, 成功获得了具有 可见光响应活性的 $\mathrm{Au} / \mathrm{Bi}_{2} \mathrm{WO}_{6}$ 异质光催化剂, 并借助 XRD、FE-SEM、HR-TEM、XPS 和 UV-Vis-DRS 谱等手段对 其物相组成、形貌和光吸收特性进行表征, 以罗丹明 $\mathrm{B}(\mathrm{RhB})$ 和苯酚为模型污染物对其光催化性能进行研究。实验 结果表明, 与纯 $\mathrm{Bi}_{2} \mathrm{WO}_{6}$ 相比, 所得 $\mathrm{Au} / \mathrm{Bi}_{2} \mathrm{WO}_{6}$ 异质纳米结构对染料降解具有较高的活性, 当 $\mathrm{Au}$ 负载量为 $1.5 \mathrm{at} \%$ 时, $\mathrm{Au} / \mathrm{Bi}_{2} \mathrm{WO}_{6}$ 复合催化剂的催化活性最好, 其光催化降解 $\mathrm{RhB}$ 和苯酚的表观速率常数分别是纯 $\mathrm{Bi}_{2} \mathrm{WO}_{6}$ 的 1.5 倍 和 2.2 倍。自由基捕获实验表明, 光生空穴 $\left(\mathrm{h}^{+}\right)$和 $\mathrm{O}_{2}^{-}$是 $\mathrm{RhB}$ 在 $\mathrm{Au} / \mathrm{Bi}_{2} \mathrm{WO}_{6}$ 催化材料上光催化降解的主要活性物种。 机理分析表明, $\mathrm{Au} / \mathrm{Bi}_{2} \mathrm{WO}_{6}$ 活性增强归因于光生电子从 $\mathrm{Bi}_{2} \mathrm{WO}_{6}$ 的导带向 $\mathrm{AuNPs}$ 表面迁移, 降低光生电子-空穴对的 复合率, 同时, $\mathrm{Au}$ NPs 的等离子共振效应(SPR)拓展了催化剂在可见光区的响应范围, 从而显著提高了 $\mathrm{Au} / \mathrm{Bi}_{2} \mathrm{WO}_{6}$ 异质光催化的剂活性。 $\mathrm{Au} N \mathrm{NP}$ 修饰 $\mathrm{Bi}_{2} \mathrm{WO}_{6}$ 异质催化剂光太阳能驱动在污水处理方面具有潜在应用。

关 键 词: $\mathrm{Au} / \mathrm{Bi}_{2} \mathrm{WO}_{6}$ 异质纳米结构; 光催化剂; 光还原法; 电子转移; 等离子共振效应(SPR)

中图分类号: 0643 文献标识码: A

\section{Au Nanoparticles (NPs) Surface Plasmon Resonance Enhanced Photocatalytic Activities of $\mathrm{Au} / \mathrm{Bi}_{2} \mathrm{WO}_{6}$ Heterogeneous Nanostructures}

\author{
WANG Dan-Jun, WANG Chan, ZHAO Qiang, GUO Li, YANG Xiao, WU Jiao, FU Feng \\ (College of Chemistry \& Chemical Engineering, Yan'an University, Shaanxi Key Laboratory of Chemical Reaction Engineering, \\ Yan'an 716000, China)
}

\begin{abstract}
Novel visible-light-responsive $\mathrm{Au} / \mathrm{Bi}_{2} \mathrm{WO}_{6}$ heterogeneous nanostructures were successfully prepared through in situ growth gold nanoparticles (Au NPs) on the second-structural nanosheets of three-dimensional (3D) $\mathrm{Bi}_{2} \mathrm{WO}_{6}$ via a facile photoreduction process. XRD, FE-SEM, HR-TEM, FT-IR, XPS, and UV-Vis-DRS spectra were employed to investigate the phase composition, morgphology and light-absorption properties of as-preapared samples. Rhodamine $\mathrm{B}(\mathrm{RhB})$ and phenol were selected as model pollutants to evaluate photocatalytic activities of samples. The experimental results reveal that the as-prepared $\mathrm{Au} / \mathrm{Bi}_{2} \mathrm{WO}_{6}$ heterogeneous nanostructures exhibit much higher photocatalytic activities than pure $\mathrm{Bi}_{2} \mathrm{WO}_{6}$ for dye degradation. It is also revealed that $1.5 \mathrm{at} \% \mathrm{Au} / \mathrm{Bi}_{2} \mathrm{WO}_{6}$ sample exhibit the best photocatalytic activities in the degradation process of $\mathrm{RhB}$ and phenol, the apparent rate constant is about 1.5 and
\end{abstract}

收稿日期: 2017-08-07; 收到修改稿日期：2017-10-29

基金项目: 国家自然科学基金(21666039，21663030); 陕西省科技厅项目(2013SZS20-P01，2015SF291); 陕西省教育厅基金 (15JS119); 陕西省大学生创新创业训练计划项目 (1517，1563); 地方高校国家级大学生创新创业训练计划项目 (12280); 延安大学研究生创新项目(YCX201733)

National Natural Science Foundation of China(21666039, 21663030); Science and Technology Department of Shaanxi Province(2013SZS20-P01, 2015SF291); Natural Science Program of Education Department of Shaanxi Province (15JS119); College Students' Innovation and Entrepreneurship Training Programs of Shaanxi Province (1517, 1563); National Local College Students' Innovation and Entrepreneurship Training Program(12280); Graduate Innovation Training Program of Yan'an University(YCX201733)

作者简介: 王丹军(1976-), 男, 教授. E-mail: wangdj761118@163.com 
2.2 times as high as that of pure $\mathrm{Bi}_{2} \mathrm{WO}_{6}$ under visible light irradiation, respectively. Moreover, the trapping experiments results show that photogenerated hole $\left(\mathrm{h}^{+}\right)$and $\cdot \mathrm{O}_{2}^{-}$serve as the main active species for the photodegradation of $\mathrm{RhB}$ over $\mathrm{Au} / \mathrm{Bi}_{2} \mathrm{WO}_{6}$ heterogeneous nanostructures. The enhanced photocatalytic efficiencies of $\mathrm{Au} / \mathrm{Bi}_{2} \mathrm{WO}_{6}$ heterogeneous nanostructures are attributed to the charge transfer from $\mathrm{Bi}_{2} \mathrm{WO}_{6}$ to the deposited $\mathrm{Au} \mathrm{NPs}$ as well as their surface plasmon resonance (SPR) absorption, which enhance the migration efficiency of the electron-holes and retard the recombination of electrons-hole pairs. Au NPs decorated $\mathrm{Bi}_{2} \mathrm{WO}_{6}$ heterogeneous nanostructures have considerable potential applications in solar-driven wastewater treatment.

Key words: $\mathrm{Au} / \mathrm{Bi}_{2} \mathrm{WO}_{6}$ heterogeneous nanostructures; photocatalyst; photo-reduction process; electron transfer; surface plasmon resonance (SPR)

铋基光催化材料以其独特的能带结构和较高的 抗光腐蚀稳定性, 在光催化领域有着广阔的应用前 景。其中, $\mathrm{Bi}_{2} \mathrm{WO}_{6}$ 是研究最为广泛的一种铋系材料, 因其具有优异的物理化学和光电性能、良好的稳定 性、低廉的成本和环境友好等性能而成为以太阳能 为光源, 利用生物质、二氧化碳和水来制取化学品 和燃料以及光催化降解环境污染物的优秀光催化材 料 ${ }^{[1-3]}$ 。然而, $\mathrm{Bi}_{2} \mathrm{WO}_{6}$ 作为光催化材料存在以下缺点: 第一, 只能被紫外区以及 $450 \mathrm{~nm}$ 以下的部分可见光 激发, 只占太阳光谱的一小部分, 可见光响应范围 较窄; 第二, 光生电子-空穴对复合率较高。这两方 面的不足严重限制了 $\mathrm{Bi}_{2} \mathrm{WO}_{6}$ 光催化体系的光转换 效率, 因此, 人们采用不同方法对 $\mathrm{Bi}_{2} \mathrm{WO}_{6}$ 进行改性 ${ }^{[4]}$, 如引入本征缺陷和掺杂 ${ }^{[5]}$, 采用微结构调控机制制 备高结晶度、大比表面积、高活性面暴露的 $\mathrm{Bi}_{2} \mathrm{WO}_{6}$ (纳 米粒子 ${ }^{[6]}$ 、一维 ${ }^{[7]}$ 、二维 ${ }^{[8-9]}$ 和三维 $\left.{ }^{[10-11]}\right)$, 采用贵金 属表面修饰 ${ }^{[12-13]}$ 和构筑异质结 ${ }^{[14-17]}$ 等策略, 抑制载 流子的复合, 并提高量子效率, 从而改善 $\mathrm{Bi}_{2} \mathrm{WO}_{6}$ 基 催化材料的性能。

贵金属纳米粒子 (NPs) 的表面等离子共振效应 成为提高光催化效率的新途径 ${ }^{[18-19]}$, 由于贵金属纳 米粒子 $(\mathrm{Ag}, \mathrm{Pt}, \mathrm{Au})$ 具有很强的可见光吸收能力, 能 够与半导体复合形成表面等离子体光催化材料。因 此, 将贵金属纳米粒子与半导体结合形成异质结构 成为广泛使用的光催化材料改性策略 ${ }^{[20-28]}$ 。例如, 人们采用 AuNPs 修饰 $\mathrm{TiO}_{2}{ }^{[20-21]} 、 \mathrm{ZnO}^{[22]} 、 \mathrm{CdS}^{[23]}$ 、 $\mathrm{BiVO}_{4}{ }^{[24]}$; 采用 $\mathrm{AgNPs}$ 修饰 $\mathrm{TiO}_{2}{ }^{[25]} 、 \mathrm{Bi}_{2} \mathrm{WO}_{6}{ }^{[26]}$, 采 用 Pt NPs 修饰 $\mathrm{Bi}_{2} \mathrm{WO}_{6}{ }^{[27]}$ 和 $\mathrm{TiO}_{2}{ }^{[28]}$ 等。

文献报道, 利用 $\mathrm{Ag}$ 和 $\mathrm{Pt}$ 纳米粒子进行表面修 饰, 可显著提高 $\mathrm{Bi}_{2} \mathrm{WO}_{6}$ 的光催化活性 ${ }^{[26-27]}$ 。近期, AuNPs 作为半导体表面敏化剂的研究引起人们的广 泛关注 ${ }^{[29-30]}$ 。本工作采用光还原技术在 $\mathrm{Bi}_{2} \mathrm{WO}_{6}$ 微 球的二级结构纳米片表面原位沉积 $\mathrm{Au}$ 纳米粒子, 得到 $\mathrm{Au} / \mathrm{Bi}_{2} \mathrm{WO}_{6}$ 异质纳米结构, 并对 $\mathrm{Au} / \mathrm{Bi}_{2} \mathrm{WO}_{6}$ 的 活性增强机理进行了初步探讨。

\section{1 实验方法}

\section{1 主要试剂}

铇酸钠, 天津市科密欧化学试剂有限公司; 硝 酸铋, 天津市致远化学试剂有限公司; 氯金酸, 上 海阿拉丁生化科技股份有限公司; 无水乙醇, 天津 市富宇精细化工有限公司; 罗丹明 $\mathrm{B}$, 天津市天新 精细化工开发中心; 硝酸, 天津市富宇精细化工有 限公司。以上药品均为分析纯试剂, 实验用水为自 制 I 级蒸馏水。

\section{2 样品的制备}

\subsection{1 $\mathrm{Bi}_{2} \mathrm{WO}_{6}$ 的制备}

准确称取 $0.97014 \mathrm{~g} \mathrm{Bi}\left(\mathrm{NO}_{3}\right)_{3} \cdot 5 \mathrm{H}_{2} \mathrm{O}$, 并将其溶 解在 $4 \mathrm{~mol} / \mathrm{L}$ 的硝酸中, 称取 $0.3299 \mathrm{~g} \mathrm{Na}_{2} \mathrm{WO}_{4}$, 将 其溶解在 $10 \mathrm{~mL}$ 水中, 在磁力搅拌 $30 \mathrm{~min}$ 后将 $\mathrm{Na}_{2} \mathrm{WO}_{4}$ 溶液逐滴加入到 $\mathrm{Bi}\left(\mathrm{NO}_{3}\right)_{3}$ 溶液中, 继续磁力 搅拌 $1 \mathrm{~h}$ 后转移到 $65 \mathrm{~mL}$ 带有聚四氟乙烯内衬的不 锈钢高压反应釜中，在 $190^{\circ} \mathrm{C}$ 下水热反应 $2 \mathrm{~h}$ 。水热 反应完毕后, 使其自然冷却至室温, 然后将所得沉 淀依次水洗、醇洗和干燥, 得到纯 $\mathrm{Bi}_{2} \mathrm{WO}_{6}$ 。

\subsection{2 $\mathrm{Au} / \mathrm{Bi}_{2} \mathrm{WO}_{6}$ 的制备}

称取 $0.3489 \mathrm{~g} \mathrm{Bi}_{2} \mathrm{WO}_{6}$ 并分散在 $10 \mathrm{~mL}$ 水中，逐 滴加入 $2.25 \mathrm{~mL}$ 浓度为 $0.001 \mathrm{~g} / \mathrm{mL}$ 的 $\mathrm{HAuCl}_{4}$ 溶液, 将上述混合溶液置于光化学反应仪中, 黑暗状态下 搅拌 $1 \mathrm{~h}$ 并通入高纯氮以除去溶解氧, 然后加入 $6 \mathrm{~mL}$ 无水乙醇和 $4 \mathrm{~mL}$ 去离子水, 并以 $300 \mathrm{~W}$ 的氙灯光 照。取上清液采用 ICP-AES 测定液体 $\mathrm{Au}$ 的含量, 待 $\mathrm{Au}$ 沉积完全后(约 $3 \mathrm{~h}$ ), 离心分离所得沉淀经水洗、 醇洗、干燥得到 $1.5 \mathrm{at} \% \mathrm{Au} / \mathrm{Bi}_{2} \mathrm{WO}_{6}$ 。改变 $\mathrm{HAuCl}_{4}$ 的量, 可得系列 $\mathrm{Au} / \mathrm{Bi}_{2} \mathrm{WO}_{6}$ 。采用电感耦合等离子 体-原子发射光谱法(ICP-AES)测定所得 $\mathrm{Au} / \mathrm{Bi}_{2} \mathrm{WO}_{6}$ 经混合酸(盐酸/硝酸)消解后 $\mathrm{Au}$ 的含量, 发现其含 量与理论沉积量基本一致。

\section{3 催化活性评价}

以 $300 \mathrm{~W}$ 氙灯(滤光片滤掉 $420 \mathrm{~nm}$ 以下的光) 作 
为可见光光源，以罗丹明 $\mathrm{B}(\mathrm{RhB})$ 和苯酚为模型污染 物, 考察 $\mathrm{Au} / \mathrm{Bi}_{2} \mathrm{WO}_{6}$ 的光催化活性。具体步骤如下: 将 $0.20 \mathrm{~g}$ 催化剂加入到装有 $200 \mathrm{~mL}$ 浓度为 $20 \mathrm{mg} / \mathrm{L}$ 的 $\mathrm{RhB}$ 溶液的石英反应瓶中, 置于光化学反应仪中并 在黑暗状态下磁力搅拌 $30 \mathrm{~min}$, 使体系达到吸附平 衡。开启光源, 每隔 $10 \mathrm{~min}$ 取样, 经离心分离催化 后，在波长为 $554 \mathrm{~nm}$ 处测定 $\mathrm{RhB}$ 的吸光度，以吸光 度换算成浓度进行定量分析。在苯酚的降解过程中, 苯酚初始浓度为 $10 \mathrm{mg} / \mathrm{L}$, 催化剂的用量和实验步 骤同上。取离心所得上清液采用 4-氨基安替比林分 光光度法测定(HJ 503-2009)苯酚的浓度。

$\mathrm{RhB}$ 和苯酚的光催化降解满足一级动力学, $\ln \left(C_{0} / C_{t}\right)=k_{\mathrm{app}} t$, 其中 $C_{0}$ 为暗反应结束后的浓度, $C_{t}$ 为不同时刻的浓度, $k$ 为表观速率常数。

\section{4 样品的表征}

用日本岛津公司 XRD-7000 型全自动 X 射线粉 末衍射仪 (XRD) 鉴定样品的物相组成, 辐射源为 $\mathrm{CuK} \alpha(\mathrm{Ni}$ 滤波片, $\lambda=0.15418 \mathrm{~nm})$, 管电压 $40 \mathrm{kV}$, 管 电流 $30 \mathrm{~mA}$, 步长 $0.02^{\circ}$, 扫描速度 $8^{\circ} \cdot \mathrm{min}^{-1}$, 扫描范 围 $2 \theta=20^{\circ} \sim 80^{\circ}$ 。采用日本电子 JEOL-6701 型场发射 扫描电子显微镜对样品的微观形貌、尺寸进行观察 与分析。采用日本电子 JEM-2100 型透射电子显微 镜对样品进行高分辨透射电镜(HR-TEM)观察。采用 美国 PE 公司 PH54500 型光电子能谱仪测试样品所 含元素的电子结合能, 铝靶 $\mathrm{Al} \mathrm{K \alpha}(1486.6 \mathrm{eV})$ 为激发 源, 用 $\mathrm{C} 1 \mathrm{~s}$ 的电子结合能 $(284.6 \mathrm{eV})$ 为标准进行误差 校正。采用日本岛津 UV-2550 型紫外-可见分光光度 计测定样品的光吸收性能，扫描范围 200 800 nm, 以标准 $\mathrm{BaSO}_{4}$ 为参比。

\section{2 结果与讨论}

\subsection{XRD 和 XPS 分析}

图 1(a)是 $190^{\circ} \mathrm{C}$ 水热反应 $2 \mathrm{~h}$ 所得 $\mathrm{Bi}_{2} \mathrm{WO}_{6}$ 和 $\mathrm{Au} / \mathrm{Bi}_{2} \mathrm{WO}_{6}$ 的 XRD 图谱, 由图可知, 纯 $\mathrm{Bi}_{2} \mathrm{WO}_{6}$ 的 在 $2 \theta=28.30^{\circ}(131) 、 32.67^{\circ}(060) 、 32.79^{\circ}(200)$ 、 $32.91^{\circ}(002) 、 47.14^{\circ}(202) 、 55.66^{\circ}(191) 、 76.07^{\circ}(193) 、$ $78.53^{\circ}(204)$ 处的特征衍射峰, 与标准卡片 (JCPDS 39-0256)一致, 表明样品为正交晶系 $\mathrm{Bi}_{2} \mathrm{WO}_{6}$ 。图 1(b) 为 $\mathrm{Au} / \mathrm{Bi}_{2} \mathrm{WO}_{6}$ 在 $300^{\circ} \mathrm{C}$ 下焙烧所得样品的 XRD 图谱, 样品在 $2 \theta=38.253^{\circ}(111) 、 44.462^{\circ}(200)$ 和 $64.695^{\circ}(220)$ 处出现了 $\mathrm{Au}$ 的特征衍射峰, 表明 $\mathrm{Au}$ 成功沉积在 $\mathrm{Bi}_{2} \mathrm{WO}_{6}$ 表面, 且 $\mathrm{Au}$ 特征衍射峰强随着沉积量的增 加而增强。

图 2 是 $1.5 \mathrm{at} \% \mathrm{Au} / \mathrm{Bi}_{2} \mathrm{WO}_{6}$ 的 XPS 图谱。由图 2(a)

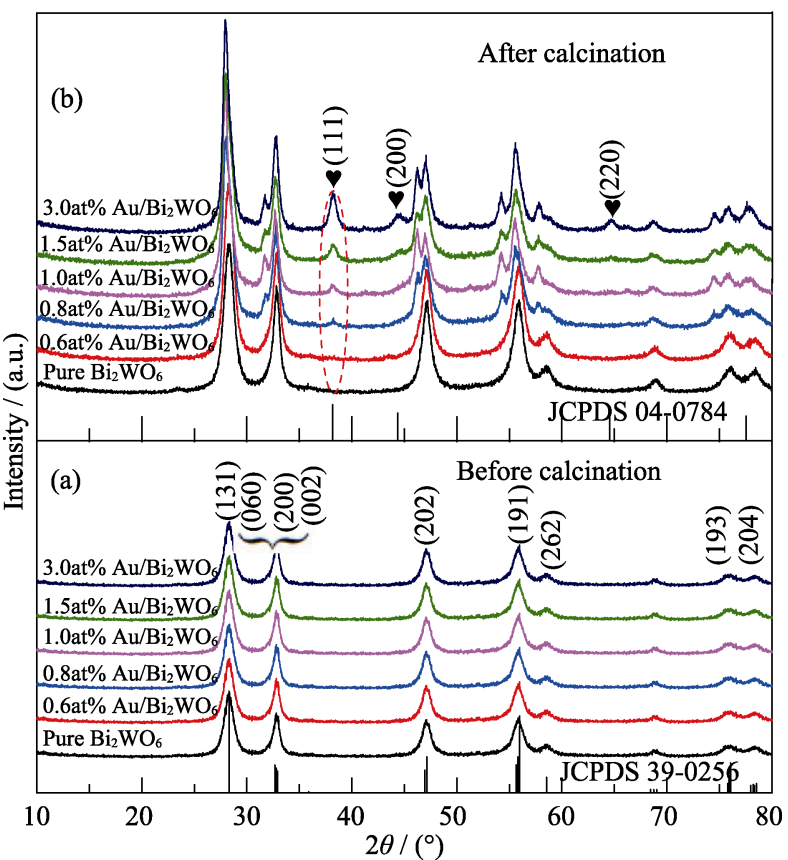

图 1 纯 $\mathrm{Bi}_{2} \mathrm{WO}_{6}$ 和 $\mathrm{Au} / \mathrm{Bi}_{2} \mathrm{WO}_{6}$ 焙烧前(a)后(b)的 XRD 图谱 Fig.1 XRD patterns of pure $\mathrm{Bi}_{2} \mathrm{WO}_{6}$ and $\mathrm{Au} / \mathrm{Bi}_{2} \mathrm{WO}_{6}$ (a) Before calcinations; (b) After calcinations
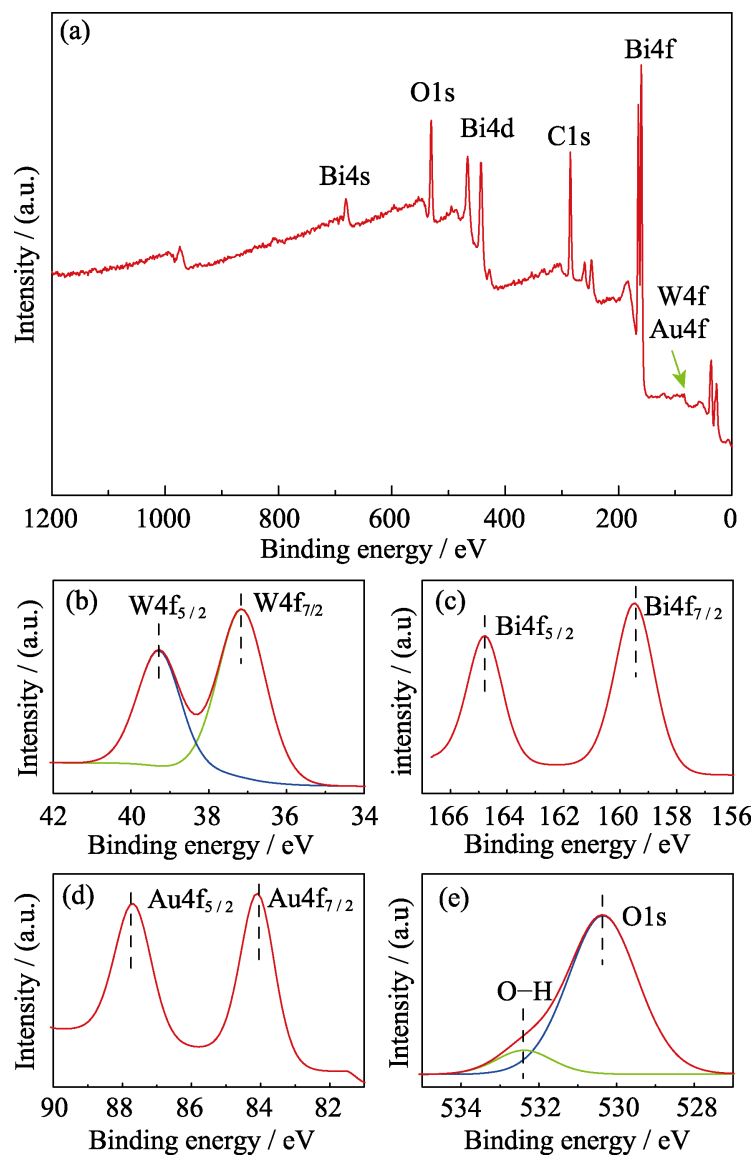

图 $21.5 \mathrm{at} \% \mathrm{Au} / \mathrm{Bi}_{2} \mathrm{WO}_{6}$ 的 XPS 图谱

Fig. 2 XPS spectra of $1.5 \mathrm{at} \% \mathrm{Au} / \mathrm{Bi}_{2} \mathrm{WO}_{6}$ sample (a) Survey; (b) W4f; (c) Bi4f; (d) Au4f; (e) O1s

可知，样品中含有 $\mathrm{Bi} 、 \mathrm{O} 、 \mathrm{~W} 、 \mathrm{C} 、 \mathrm{Au}$, 其中 $\mathrm{C}$ 元素 归属于仪器本身的 C 污染。图 2(b) (e) 分别为 W4f、 
Bi4f、Au1s、O1s 相应的高分辨 XPS 图谱, 图 2(b) 中, 结合能为 37.24 和 $39.26 \mathrm{eV}$ 的特征峰对应于 $\mathrm{W} 4 \mathrm{f}_{7 / 2}$ 和 $\mathrm{W} 4 \mathrm{f}_{5 / 2}$; 图 2(c)中, 结合能为 159.60 和 $164.90 \mathrm{eV}$ 的特征峰分别对应于 $\mathrm{Bi}_{4 / 2}$ 和 $\mathrm{Bi}_{4} \mathrm{f}_{5 / 2}$; 图 2(d) 中, 结合能为 84.02 和 $87.84 \mathrm{eV}$ 的特征峰分别对应 于 $\mathrm{Au} 4 \mathrm{f}_{7 / 2}$ 和 $\mathrm{Au} 4 \mathrm{f}_{5 / 2}$, 表明 $\mathrm{Au}$ 成功地沉积在 $\mathrm{Bi}_{2} \mathrm{WO}_{6}$ 表面; 图 2(e)中, 结合能为 530.18 和 $532.25 \mathrm{eV}$ 分别对 应于晶格氧和表面羟基氧 ${ }^{[17]}$ 。

\subsection{SEM 和 TEM/HR-TEM 分析}

图 3(a) (f) 是样品的 FE-SEM 电镜照片, 由图可 见, $\mathrm{Bi}_{2} \mathrm{WO}_{6}$ 是由厚度为 $10 \sim 20 \mathrm{~nm}$ 的纳米片组装而
成的三维球形结构, 直径为 $2 \sim 3 \mu \mathrm{m} ; \mathrm{Au} / \mathrm{Bi}_{2} \mathrm{WO}_{6}$ 与 纯的 $\mathrm{Bi}_{2} \mathrm{WO}_{6}$ 样品的形貌基本一致。从图 3(f) 可以看 到有大量 $\mathrm{Au}$ 纳米粒子沉积在 $\mathrm{Bi}_{2} \mathrm{WO}_{6}$ 纳米片上。图 4 为样品的 TEM 和 HR-TEM 照片, 从图 4(a) (b) 可以 看出, 纯 $\mathrm{Bi}_{2} \mathrm{WO}_{6}$ 是由大量纳米片组装而成; 由图 4(c) 可以看出, 相邻两晶面的距离为 $0.314 \mathrm{~nm}$, 与 $\mathrm{Bi}_{2} \mathrm{WO}_{6}$ 的 (131) 晶面间距相一致。图 4(d) (f) 为 $1.5 \mathrm{at} \% \mathrm{Au} / \mathrm{Bi}_{2} \mathrm{WO}_{6}$ 的 TEM/HR-TEM 照片, 由图可以 看出, 大量尺寸约为 $10 \mathrm{~nm}$ 的 $\mathrm{Au}$ 纳米粒子均匀分散 在 $\mathrm{Bi}_{2} \mathrm{WO}_{6}$ 纳米片的表面; $0.235 \mathrm{~nm}$ 晶面间距对应于 $\mathrm{Au}$ 的(111)面。
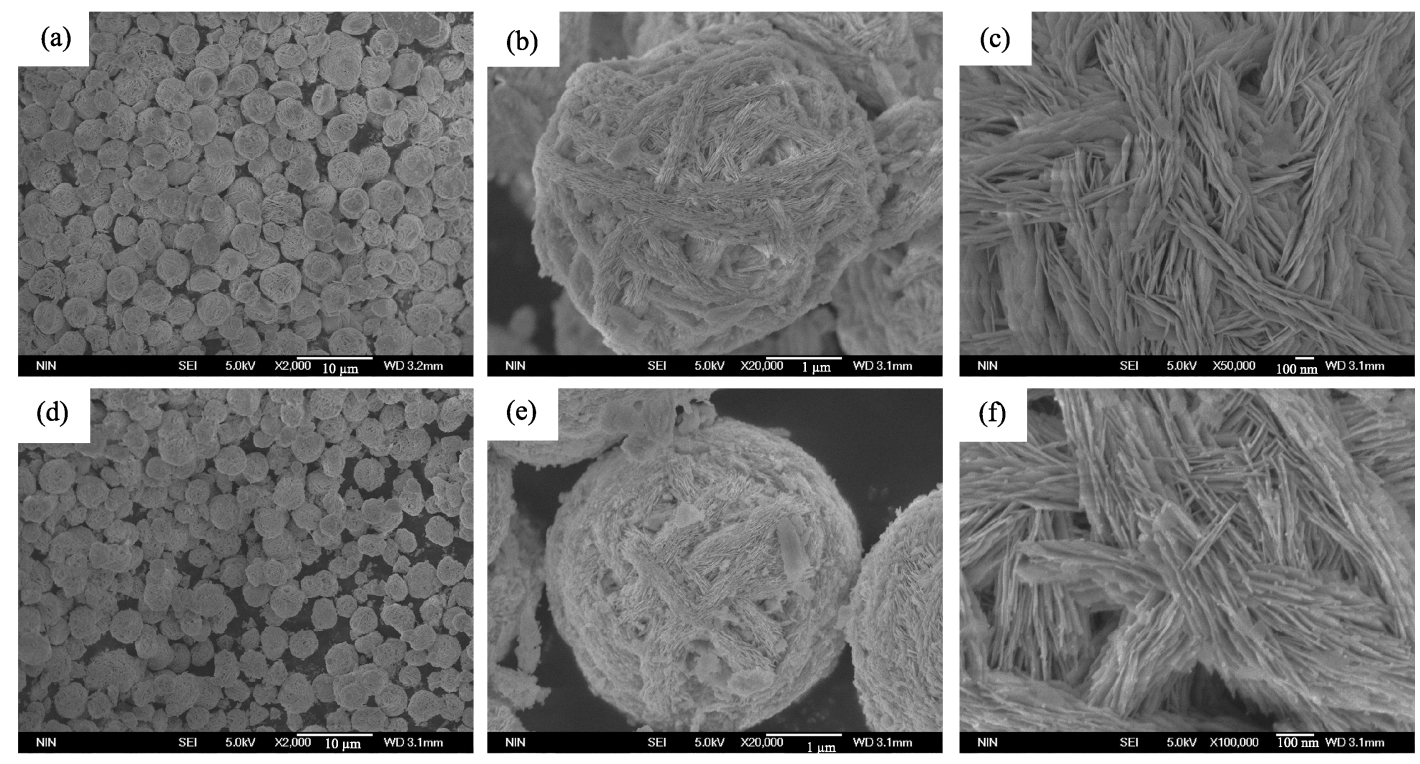

图 3 光催化剂的 FE-SEM 电镜照片

Fig. 3 High magnification SEM images of as-prepared samples (a)-(c) Pure $\mathrm{Bi}_{2} \mathrm{WO}_{6}$; (d)-(f) $1.5 \mathrm{at} \% \mathrm{Au} / \mathrm{Bi}_{2} \mathrm{WO}_{6}$
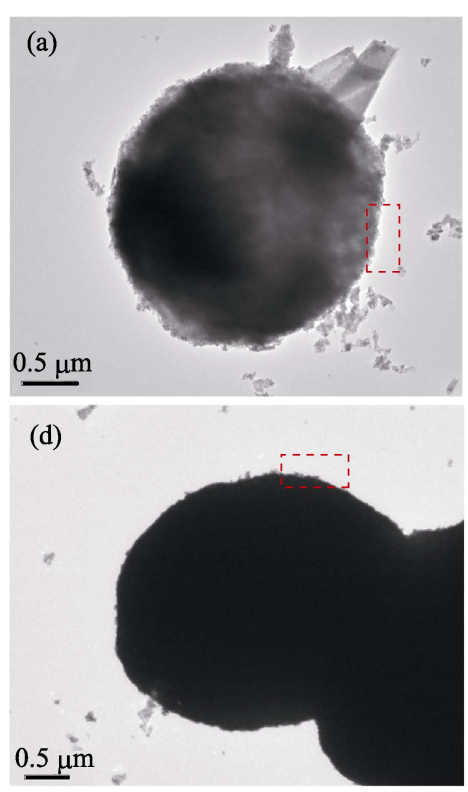
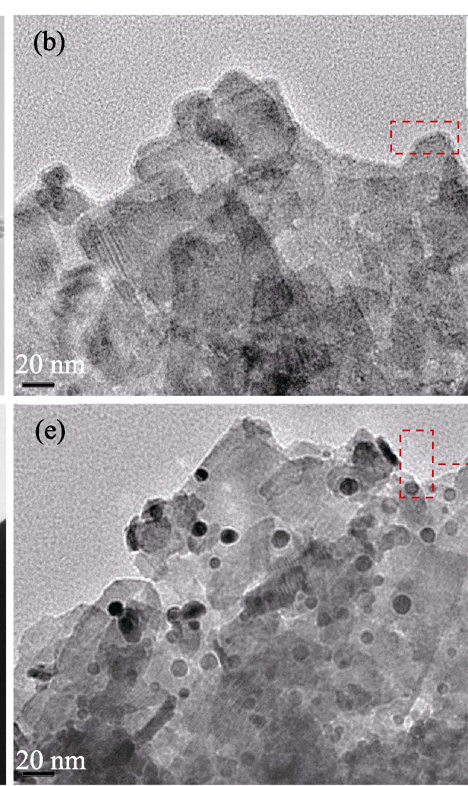
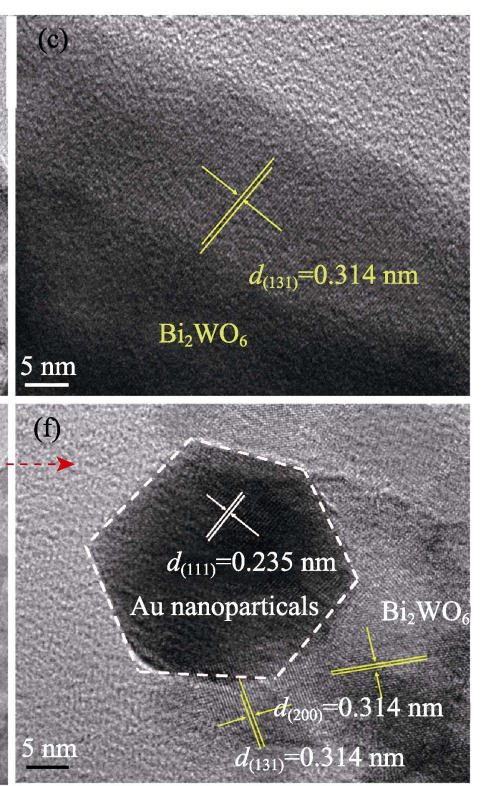

图 4 (a) (c) 纯 $\mathrm{Bi}_{2} \mathrm{WO}_{6}$ 和(d) (f) $1.5 \mathrm{at} \% \mathrm{Au} / \mathrm{Bi}_{2} \mathrm{WO}_{6}$ 异质纳米结构的 TEM/HR-TEM 电镜照片

Fig. 4 TEM/HR-TEM images of (a)-(c) pure $\mathrm{Bi}_{2} \mathrm{WO}_{6}$ and (d)-(f) $1.5 \% \mathrm{Au} / \mathrm{Bi}_{2} \mathrm{WO}_{6}$ heterogeneous nanostructures 


\section{3 光吸收特性}

图 5 是合成的系列光催化剂的固体紫外-可见漫 反射(UV-Vis-DRS)谱图, 从图中可以看出, 各样品 在 300 400 nm 处均有较强的吸收, 与纯 $\mathrm{Bi}_{2} \mathrm{WO}_{6}$ 相 比, $\mathrm{Au} / \mathrm{Bi}_{2} \mathrm{WO}_{6}$ 复合光催化剂在可见光区有明显的 吸收, 出现一个大包峰。半导体的带隙可以由下列 公式计算: $(\alpha \mathrm{h} v)^{1 / n}=A\left(\mathrm{~h} v-E_{\mathrm{g}}\right)$, 式中: $\alpha 、 \mathrm{~h} 、 v 、 A$ 和 $E_{\mathrm{g}}$ 分别表示吸收系数、普朗克常数、光子频率、常 数和带隙能量。 $\mathrm{Bi}_{2} \mathrm{WO}_{6}$ 属于直接跃迁半导体, 将 $n=0.5$ 代入进行计算。根据计算结果, $\mathrm{Bi}_{2} \mathrm{WO}_{6}$ 的带隙 约为 $2.81 \mathrm{eV}, 1.5 \mathrm{at} \% \mathrm{Au} / \mathrm{Bi}_{2} \mathrm{WO}_{6}$ 的带隙与纯 $\mathrm{Bi}_{2} \mathrm{WO}_{6}$ 几乎相同, 约为 $2.72 \mathrm{eV}$ 。从图 5 可以看出, $\mathrm{Au} / \mathrm{Bi}_{2} \mathrm{WO}_{6}$ 催化剂在 $580 \mathrm{~nm}$ 处有一宽的强吸收峰, 可归属于 $\mathrm{Au}$ 纳米粒子的等离子共振吸收(SPR), 表 明 $\mathrm{Au}$ 纳米粒子表面沉积有利于拓宽催化剂在可见 光区的光吸收范围。

\section{4 可见光照射下光催化剂的活性探究}

由图 6(a)可以看出, $\mathrm{Au} / \mathrm{Bi}_{2} \mathrm{WO}_{6}$ 作为光催化剂时, 可见光照射 $50 \mathrm{~min}$, 初始浓度为 $20 \mathrm{mg} / \mathrm{L}$ 的 $\mathrm{RhB}$ 的 降解率高达 $73 \%$, 而纯 $\mathrm{Bi}_{2} \mathrm{WO}_{6}$ 作为光催化剂时, $\mathrm{RhB}$ 的降解率只有 $40 \%$ 。 RhB 的光催化降解符合一
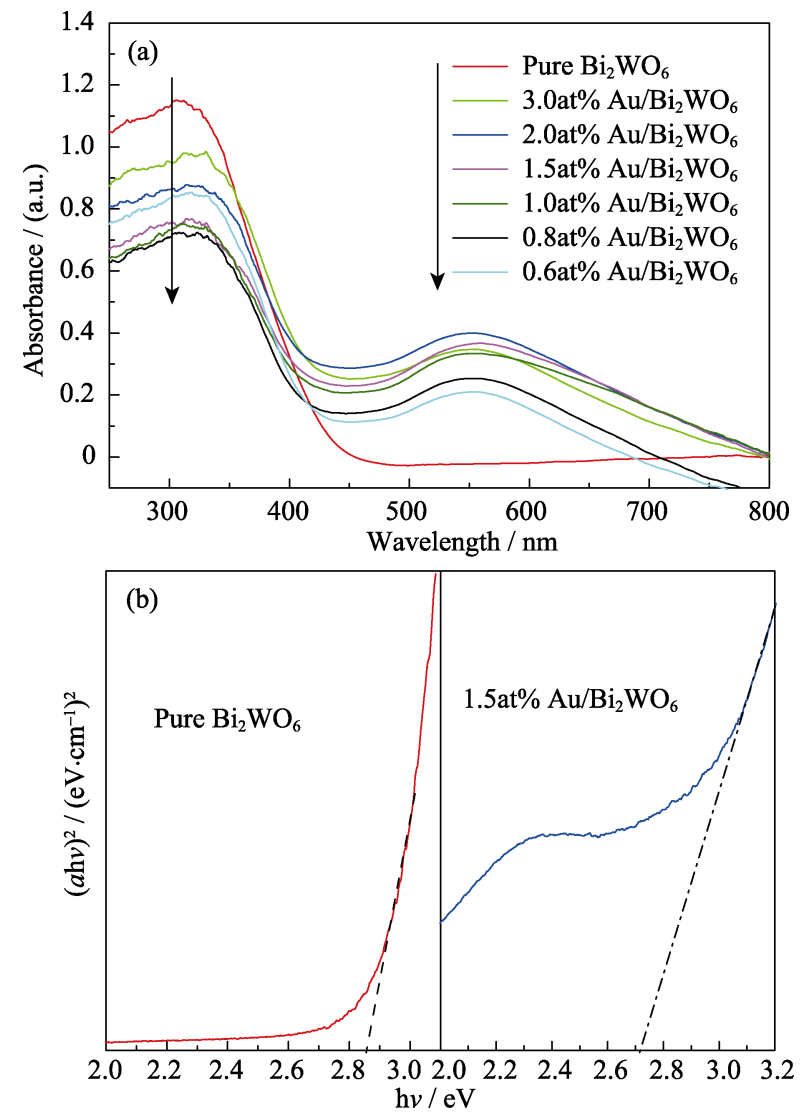

图 $5 \mathrm{Au} / \mathrm{Bi}_{2} \mathrm{WO}_{6}$ 的 UV-Vis-DRS 图谱

Fig. 5 UV-Vis absorbance spectra of $\mathrm{Au} / \mathrm{Bi}_{2} \mathrm{WO}_{6}$ heterogeneous nanostructures the $\mathrm{Au} / \mathrm{Bi}_{2} \mathrm{WO}_{6}$ sample
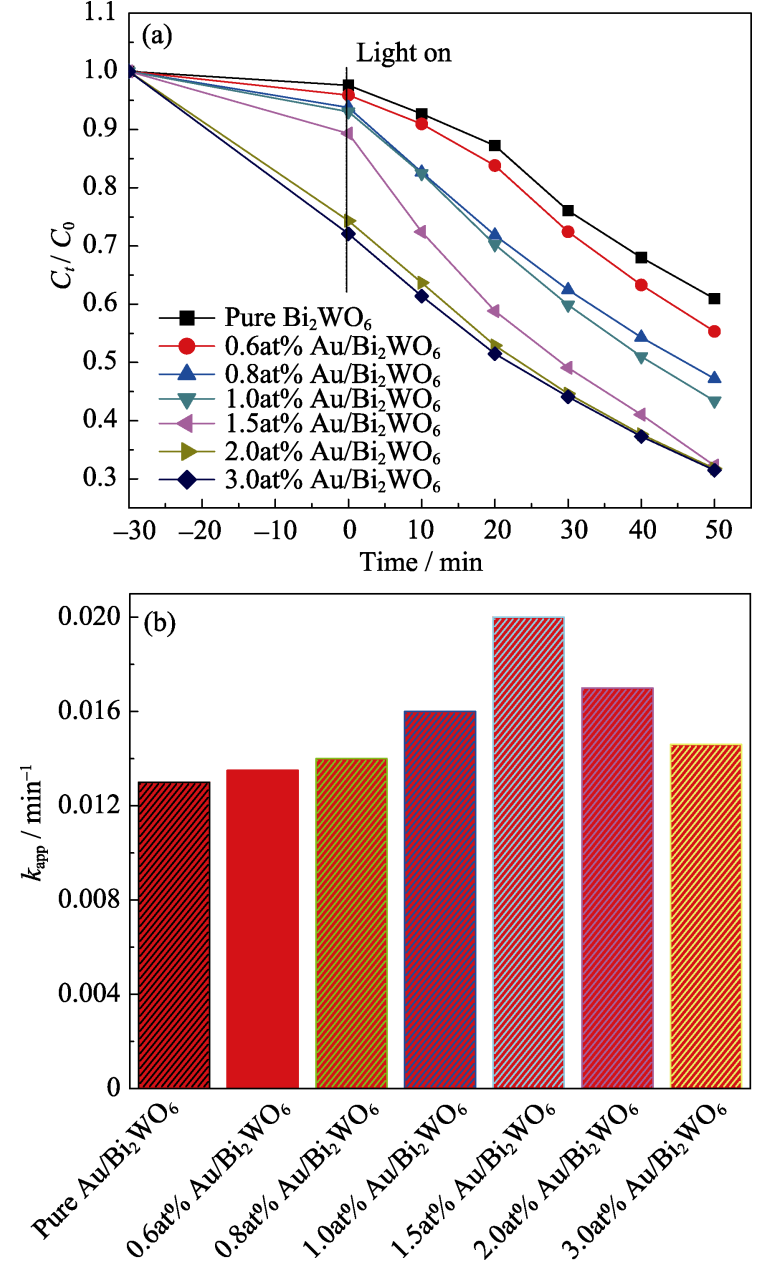

图 6 (a)不同 $\mathrm{Au} / \mathrm{Bi}_{2} \mathrm{WO}_{6}$ 催化剂降解 $\mathrm{RhB}$ 的活性和(b) $\mathrm{Au} / \mathrm{Bi}_{2} \mathrm{WO}_{6}$ 催化剂降解 $\mathrm{RhB}$ 的表观速率常数比较

Fig. 6 (a) Photocatalytic degradation acitvities and (b) apparent rate constant $\left(k_{\text {app }}\right)$ for the degradation of different $\mathrm{Au} / \mathrm{Bi}_{2} \mathrm{WO}_{6}$ photocatalysts on $\mathrm{RhB}$

级动力学模型, 如图 6(b)所示。由图可见, 表面沉积 $\mathrm{Au}$ 纳米粒子可显著提高 $\mathrm{Bi}_{2} \mathrm{WO}_{6}$ 对 $\mathrm{RhB}$ 的光催化降 解活性, 且随着 $\mathrm{Au}$ 沉积量的增加, 光催化降解活性 先增加后减小, 当 $\mathrm{Au}$ 的沉积量为 $1.5 \mathrm{at} \%$ 时, 光催化 降解活性最高, 它对 $\mathrm{RhB}$ 光催化降解的表观速率常 数 $\left(k_{\mathrm{app}} / \mathrm{min}^{-1}\right)$ 为 $0.020 \mathrm{~min}^{-1}$, 约是纯 $\mathrm{Bi}_{2} \mathrm{WO}_{6}$ 的 1.5 倍，表明 AuNPs 表面沉积可以明显提高 $\mathrm{Bi}_{2} \mathrm{WO}_{6}$ 的 光催化活性。

图 7 是苯酚的光催化降解动力学曲线。由图 7(a) 可见, 随着光照时间延长, 苯酚溶液 (显色后)在 $510 \mathrm{~nm}$ 处的吸收峰不断降低。由图 7(b)可以看出, $1.5 \mathrm{at} \% \mathrm{Au} / \mathrm{Bi}_{2} \mathrm{WO}_{6}$ 降解苯酚的表观速率常数 $k_{\mathrm{app}}$ 约 为是纯 $\mathrm{Bi}_{2} \mathrm{WO}_{6}$ 的 2.2 倍。

\section{5 光催化机理}

采用乙二胺四乙酸二钠(EDTA-2Na)、苯醌(BQ) 和异丙醇(IPA)分别作为 $\mathrm{h}^{+} 、 \mathrm{O}_{2}^{-}$和 $\cdot \mathrm{OH}$ 的捕获剂, 在 $20 \mathrm{mg} / \mathrm{L}$ 的 $\mathrm{RhB}$ 溶液中添加等浓度、等体积的捕获 

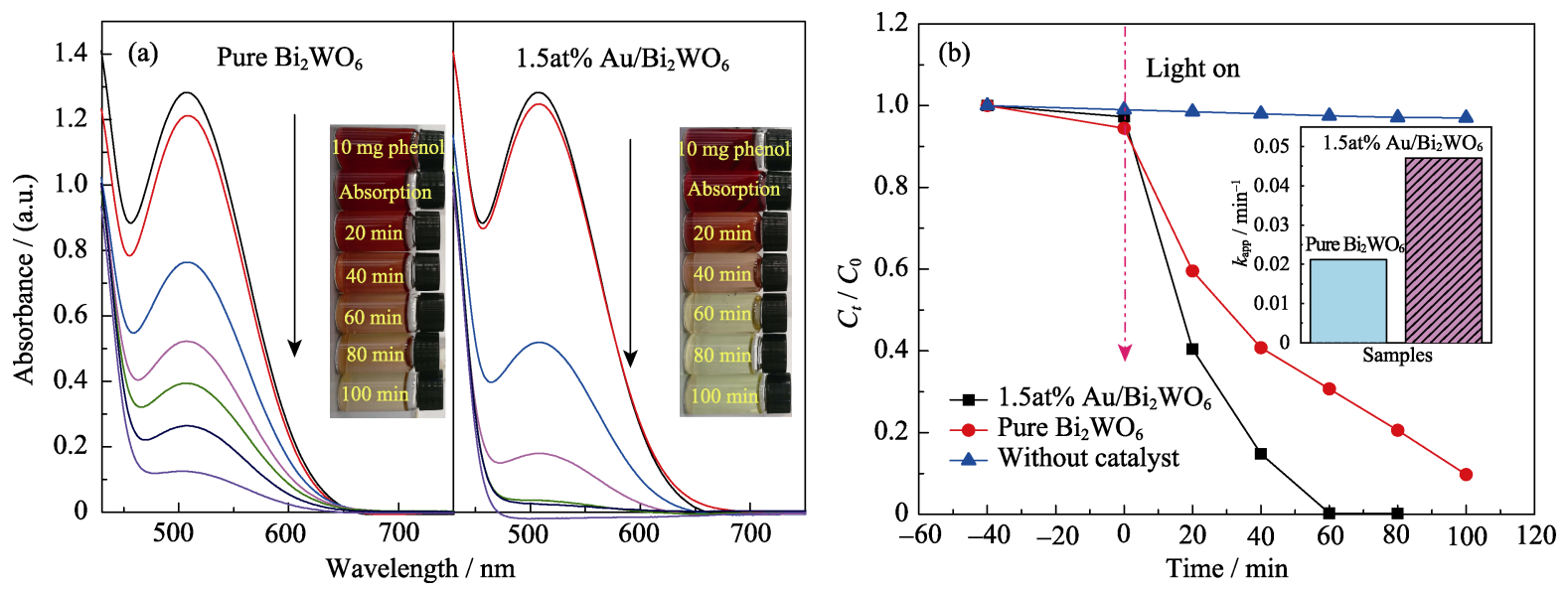

图 7 (a)不同 $\mathrm{Au} / \mathrm{Bi}_{2} \mathrm{WO}_{6}$ 催化剂降解苯酚的活性和(b) $\mathrm{Au} / \mathrm{Bi}_{2} \mathrm{WO}_{6}$ 催化剂降解苯酚的表观速率常数比较

Fig. 7 (a) Photocatalytic acitvities of $\mathrm{Bi}_{2} \mathrm{WO}_{6}$ and $1.5 \mathrm{at} \% \mathrm{Au} / \mathrm{Bi}_{2} \mathrm{WO}_{6}$ photocatalysts and (b) apparent rate constant $\left(k_{\text {app }}\right)$ for the degradation over $\mathrm{Bi}_{2} \mathrm{WO}_{6}$ and $1.5 \mathrm{at} \% \mathrm{Au} / \mathrm{Bi}_{2} \mathrm{WO}_{6}$ against phenol

剂, 以考察捕获剂 RhB 降解的影响, 所得结果如图 8 所示。从图 8 可以看出, 加入捕获剂时由于溶液的 相互稀释导致 $\mathrm{RhB}$ 的起始浓度为 $10 \mathrm{mg} / \mathrm{L}$, 没有捕 获剂存在时连续光照 $50 \mathrm{~min}, \mathrm{RhB}$ 的降解率为 $90 \%$; 加入捕获剂 EDTA-2Na 和 BQ 时, 连续光照 $50 \mathrm{~min}$, $\mathrm{RhB}$ 的降解率分别下降至 33\%和 78\%; 加入. $\mathrm{OH}$ 捕 获剂 IPA 时, RhB 的降解率变化不明显, 这表明 $\mathrm{h}^{+}$ 和. $\mathrm{O}_{2}$ - 是 $\mathrm{RhB}$ 光催化降解过程中的主要活性物种。

光催化剂的活性与其能带结构密切相关, 根据 公式 $E_{\mathrm{CB}}=X-E^{\mathrm{C}}-0.5 E_{\mathrm{g}}$ 和 $E_{\mathrm{VB}}=E_{\mathrm{CB}}+E_{\mathrm{g}}$ 计算催化剂的 价带 $(\mathrm{VB})$ 和导带 $(\mathrm{CB})$ 位置, 其中, $X$ 为 $\mathrm{Bi}_{2} \mathrm{WO}_{6}$ 的电 负性, $E^{\mathrm{C}}$ 为氢的自由电子能量。经计算可得, $\mathrm{Bi}_{2} \mathrm{WO}_{6}$ 的导带和价带分别为 $-1.04 \mathrm{eV}$ 和 $1.77 \mathrm{eV}$ 。可将光照 射下 $\mathrm{Bi}_{2} \mathrm{WO}_{6}$ 的价带电子被激发至导带, 由于 $\mathrm{Bi}_{2} \mathrm{WO}_{6}$ 的价带电势 $(1.77 \mathrm{eV})$ 小于 $\cdot \mathrm{OH} / \mathrm{H}_{2} \mathrm{O}$ 的还原电势 $(2.30 \mathrm{eV}$,

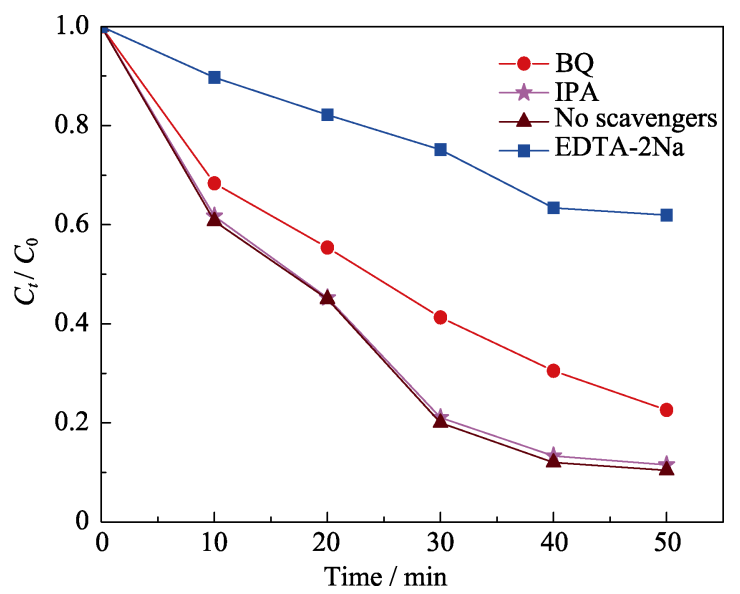

图 8 可见光照射下 $1.5 \mathrm{at} \% \mathrm{Au} / \mathrm{Bi}_{2} \mathrm{WO}_{6}$ 光催化降解 $\mathrm{RhB}$ 的 活性物种捕获实验

Fig. 8 Trapping experiments of active species in the system of photodegradation of $\mathrm{RhB}$ by $1.5 \% \mathrm{Au} / \mathrm{Bi}_{2} \mathrm{WO}_{6}$ under visible light irradiation vs. NHE), 因此光照下 $\mathrm{Bi}_{2} \mathrm{WO}_{6}$ 产生的光生空穴 $\left(\mathrm{h}^{+}\right)$ 不能将 $\mathrm{H}_{2} \mathrm{O}$ 氧化为 $\mathrm{OH}$; 由于 $\mathrm{Bi}_{2} \mathrm{WO}_{6}$ 的导带电势 $(-1.04 \mathrm{eV})$ 低于 $\mathrm{O}_{2} / \cdot \mathrm{O}_{2}^{-}$, 故其导带电子可将 $\mathrm{O}_{2}$ 还原 为 $\cdot \mathrm{O}_{2}{ }^{-}$自由基 ${ }^{[17]}$, 这与活性物种捕获实验结果一致。

当 $\mathrm{Au}$ 纳米粒子沉积在 $\mathrm{Bi}_{2} \mathrm{WO}_{6}$ 表面时, 一方面, $\mathrm{Au}$ 纳米粒子可以作为电子的“咜存器”, 抑制光生电 子和空穴对的复合，从而提高 $\mathrm{Bi}_{2} \mathrm{WO}_{6}$ 表面载流子的 分离效率。由于 $\mathrm{Au}$ 的费米能级低于 $\mathrm{Bi}_{2} \mathrm{WO}_{6}$ 的导带 边缘 ${ }^{[31]}$, 在 $\mathrm{Au} / \mathrm{Bi}_{2} \mathrm{WO}_{6}$ 异质结中, $\mathrm{Au}$ 纳米粒子和 $\mathrm{Bi}_{2} \mathrm{WO}_{6}$ 表面的相互作用会建立起表面电荷的平衡。 可见光照射下, 当光子能量大于带隙 $\left(E_{\mathrm{g}}\right)$ 时, $\mathrm{Bi}_{2} \mathrm{WO}_{6}$ 的价带 $(\mathrm{VB})$ 电子被激发至导带形成导带电子 $\left(\mathrm{e}_{\mathrm{CB}}{ }^{-}\right)$, 同时在价带留下空穴 $\left(\mathrm{h}_{\mathrm{VB}}{ }^{+}\right)$。在 $\mathrm{Au}$ 和 $\mathrm{Bi}_{2} \mathrm{WO}_{6}$ 的界面 上, 光生电子重新分配, 部分电子转移至 $\mathrm{Au}$ 纳米粒 子直至建立新的表面电荷平衡。聚集在 $\mathrm{Au}$ 纳米粒子 上的电子提升了 $\mathrm{Au}$ 纳米粒子的费米能级 $\left(E_{\mathrm{f}}\right)$, 使其 变得更负 $\left(E_{\mathrm{f}}^{*}\right)$ 并最终接近 $\mathrm{Bi}_{2} \mathrm{WO}_{6}$ 的导带(图 9)。电 荷的这种转移过程有利于提高光生电子和空穴的分 离效率，从而提高 $\mathrm{Bi}_{2} \mathrm{WO}_{6}$ 载流子的寿命 ${ }^{[32]}$ 。另一方 面, 沉积在 $\mathrm{Bi}_{2} \mathrm{WO}_{6}$ 表面的 $\mathrm{Au}$ 纳米粒子所产生的等 离子共振效应(SPR) 也会提高光催化的效率。在 $\mathrm{Au} / \mathrm{Bi}_{2} \mathrm{WO}_{6}$ 催化剂中, $\mathrm{Au}$ 纳米粒子的 SPR 效应导致 催化剂在 $580 \mathrm{~nm}$ 处有一宽的强吸收峰, 表明 $\mathrm{Au}$ 纳 米粒子的 SPR 效应显著拓宽了催化剂在可见光区的 响应范围。

结合 $\mathrm{RhB}$ 光催化降解和活性物种捕获实验结果, $\mathrm{Au} / \mathrm{Bi}_{2} \mathrm{WO}_{6}$ 的活性增强机理如下(图 9): $\mathrm{Au}$ 纳米粒子 沉积在 $\mathrm{Bi}_{2} \mathrm{WO}_{6}$ 的表面, SPR 效应可以有效拓宽 $\mathrm{Au} / \mathrm{Bi}_{2} \mathrm{WO}_{6}$ 对可见光的范围。此外, 光照下 $\mathrm{Au}$ 纳米 粒子的 SPR 效应导致光生电子从 $\mathrm{Bi}_{2} \mathrm{WO}_{6}$ 的导带向 $\mathrm{Au}$ 纳米粒子移动, 且 $\mathrm{Au}$ 纳米粒子与 $\mathrm{Bi}_{2} \mathrm{WO}_{6}$ 表面 


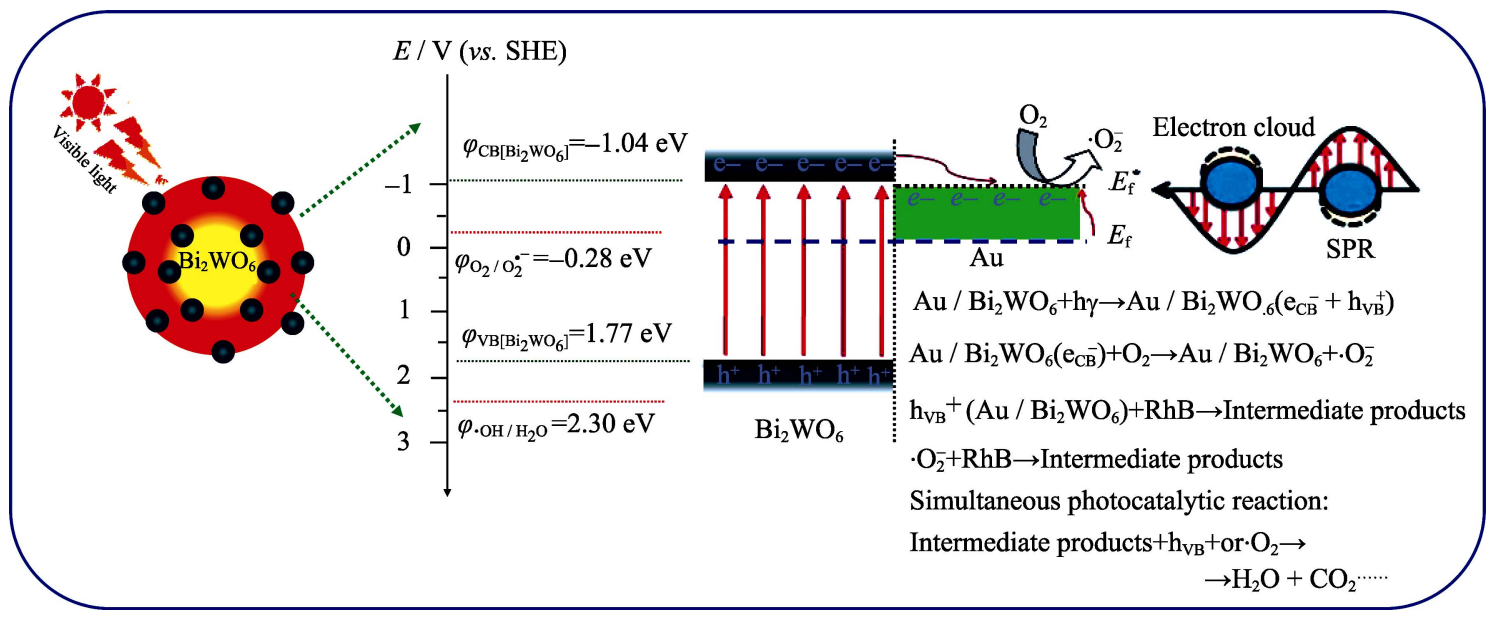

图 9 可见光照射下 $\mathrm{Au} / \mathrm{Bi}_{2} \mathrm{WO}_{6}$ 的能带结构和光可见光照射下光催化降解 $\mathrm{RhB}$ 的机理图

Fig. 9 Schematic illustration of the detailed energy alignment in the $\mathrm{Au} / \mathrm{Bi}_{2} \mathrm{WO}_{6}$ hegerogeneous nanostructure and the proposed mechanism of photocatalytic degradation of $\mathrm{RhB}$ by $\mathrm{Au} / \mathrm{Bi}_{2} \mathrm{WO}_{6}$ under visible light irradiation

建立的界面电场有利于光生电子/空穴对的分离和 迁移，而迁移至催化剂表面的光生电子被 $\mathrm{O}_{2}$ (电子 受体)捕获，形成具有氧化能力的 $\cdot \mathrm{O}_{2}^{-}$，产生的 $\cdot \mathrm{O}_{2}^{-}$ 和光生空穴作为主要的活性物种和 $\mathrm{RhB}$ 分子发生 反应，使其分解为 $\mathrm{CO}_{2}$ 和 $\mathrm{H}_{2} \mathrm{O}$ 等小分子。

\section{3 结论}

1)与纯 $\mathrm{Bi}_{2} \mathrm{WO}_{6}$ 相比, 实验制备的 $\mathrm{Au} / \mathrm{Bi}_{2} \mathrm{WO}_{6}$ 异质纳米结构对 $\mathrm{RhB}$ 和苯酚降解活性显著增强, 当 $\mathrm{Au}$ 负载量为 $1.5 \mathrm{at} \%$ 时, $\mathrm{Au} / \mathrm{Bi}_{2} \mathrm{WO}_{6}$ 的催化活性最好, 其降解 $\mathrm{RhB}$ 和苯酚的表观速率常数分别是纯 $\mathrm{Bi}_{2} \mathrm{WO}_{6}$ 的 1.5 倍和 2.2 倍。

2)自由基捕获实验表明, 光生空穴 $\left(\mathrm{h}^{+}\right)$和 $\cdot \mathrm{O}_{2}^{-}$是 $\mathrm{RhB}$ 和苯酚在 $\mathrm{Au} / \mathrm{Bi}_{2} \mathrm{WO}_{6}$ 上光催化降解的主要活性 物种。

3) $\mathrm{Au} / \mathrm{Bi}_{2} \mathrm{WO}_{6}$ 活性增强归因于光生电子从 $\mathrm{Bi}_{2} \mathrm{WO}_{6}$ 向 AuNPs 表面迁移, 有效抑制了光生电子空穴对的复合, 并且 $\mathrm{Au}$ NPs 等离子共振效应拓展 了催化剂在可见光区的响应范围, 从而显著提高了 $\mathrm{Au} / \mathrm{Bi}_{2} \mathrm{WO}_{6}$ 异质光催化剂活性。

\section{参考文献:}

[1] KONG X Y, CHOO Y Y, CHAI S P, et al. Oxygen vacancy induced $\mathrm{Bi}_{2} \mathrm{WO}_{6}$ for the realization of photocatalytic $\mathrm{CO}_{2}$ reduction over the full solar spectrum: from the UV to the NIR region. Chem. Commun., 2016, 52(99): 14242-14245.

[2] LI M, ZHANG L, FAN X, et al. Highly selective $\mathrm{CO}_{2}$ photoreduction to $\mathrm{CO}$ over $\mathrm{g}-\mathrm{C}_{3} \mathrm{~N}_{4} / \mathrm{Bi}_{2} \mathrm{WO}_{6}$ composites under visible light. $J$. Mater. Chem. A, 2015, 3(9): 5189-5196.

[3] ZHANG N, CIRIMINNA R, PAGLIARO M, et al. Nanochemistryderived $\mathrm{Bi}_{2} \mathrm{WO}_{6}$ nanostructures: towards production of sustainable chemicals and fuels induced by visible light. Chem. Soc. Rev., 2014,
43(15): 5276-5287.

[4] LI X, YU J, JARONIEC M. Hierarchical photocatalysts. Chem. Soc. Rev., 2016, 45(9): 2603-2636.

[5] XU Y, SONG J, CHEN F, et al. Amorphous Ti(IV)-modified $\mathrm{Bi}_{2} \mathrm{WO}_{6}$ with enhanced photocatalytic performance. RSC Adv., 2016, 6(70): 65902-65910.

[6] SUN S, WANG W, ZHANG L. $\mathrm{Bi}_{2} \mathrm{WO}_{6}$ Quantum dots decorated reduced graphene oxide: improved charge separation and enhanced photoconversion efficiency. J. Phys. Chem. C, 2013, 117(18): $9113-$ 9120.

[7] LIU S J, HOU Y F, ZHENG S L, et al. One-dimensional hierarchical $\mathrm{Bi}_{2} \mathrm{WO}_{6}$ hollow tubes with porous walls: synthesis and photocatalytic property. CrystEngComm., 2013, 15(20): 41244130.

[8] ZHOU Y, ZHANG Y, LIN M, et al. Monolayered $\mathrm{Bi}_{2} \mathrm{WO}_{6}$ nanosheets mimicking heterojunction interface with open surfaces for photocatalysis. Nat. Commun., 2015, 6: 8340.

[9] ZHANG L, BAHNEMANN D. Synthesis of nanovoid $\mathrm{Bi}_{2} \mathrm{WO}_{6} 2 \mathrm{D}$ ordered arrays as photoanodes for photoelectrochemical water splitting. ChemSusChem, 2013, 6(2): 283-290.

[10] ZHOU Y X, TONG L, ZENG X H, et al. Green synthesis of flower-like $\mathrm{Bi}_{2} \mathrm{WO}_{6}$ microspheres as a visible-light-driven photocatalyst. New J. Chem., 2014, 38(5): 1973-1979.

[11] SUN S, WANG W, ZHANG L. Facile preparation of threedimensionally ordered macroporous $\mathrm{Bi}_{2} \mathrm{WO}_{6}$ with high photocatalytic activity. J. Mater. Chem., 2012, 22(36): 19244-29249.

[12] WANG F, CAO K, WU Y, et al. Interfacial properties of the enhanced visible-light plasmonic $\mathrm{Ag} / \mathrm{Bi}_{2} \mathrm{WO}_{6}$ (001) nanocomposite. Appl. Surf. Sci., 2016, 360: 1075-1079.

[13] MOHAMED R M, AAZAM E S. Enhancement of photocatalytic properties of $\mathrm{Bi}_{2} \mathrm{WO}_{6}$ nanoparticles by Pt deposition. Mater. Res. Bull., 2013, 48(9): 3572-3578.

[14] MA D, WU J, GAO M, et al. Fabrication of Z-scheme $\mathrm{g}-\mathrm{C}_{3} \mathrm{~N}_{4} / \mathrm{RGO} / \mathrm{Bi}_{2} \mathrm{WO}_{6}$ photocatalyst with enhanced visible-light photocatalytic activity. Chem. Eng. J., 2016, 290: 136-146.

[15] XIAO X, WEI J, YANG Y, et al. Photoreactivity and mechanism of g- $\mathrm{C}_{3} \mathrm{~N}_{4}$ and $\mathrm{Ag}$ co-modified $\mathrm{Bi}_{2} \mathrm{WO}_{6}$ microsphere under visible light irradiation. ACS Sustain. Chem. Eng., 2016, 4(6): 30173023.

[16] JO W K, LEE J Y, NATARAJAN T S. Fabrication of hierarchically structured novel redox-mediator-free $\mathrm{ZnIn}_{2} \mathrm{~S}_{4}$ marigold flower $/ \mathrm{Bi}_{2} \mathrm{WO}_{6}$ 
flower-like direct Z-scheme nanocomposite photocatalysts with superior visible light photocatalytic efficiency. Phys. Chem. Chem. Phys., 2016, 18(2): 1000-1016.

[17] WANG D J, ZHEN Y Z, XUE G L, et al. AgBr quantum dots decorated mesoporous $\mathrm{Bi}_{2} \mathrm{WO}_{6}$ architectures with enhanced photocatalytic activities for methylene blue. J. Mater. Chem. A, 2014, 2: 11716-11727.

[18] LIU S, XU Y J. Photo-induced transformation process at gold clusterssemiconductor interface: implications for the complexity of gold clusters-based photocatalysis. Sci. Rep., 2016, 6: 22742-1-13.

[19] MA X C, DAI Y, YU L, et al. Energy transfer in plasmonic photocatalytic composites. Light Sci. Appl., 2016, 5: e6017-1-13.

[20] ZHANG G, ZHAO Z, TAN H, et al. Hierarchical $\mathrm{TiO}_{2}$ spheres decorated with $\mathrm{Au}$ nanoparticles for visible light hydrogen production. RSC Adv., 2015, 5(27): 21237-21241.

[21] WANG H, YOU T, SHI W, et al. $\mathrm{Au} / \mathrm{TiO}_{2} / \mathrm{Au}$ as a plasmonic coupling photocatalyst. J. Phys. Chem. C, 2012, 116(10): 6490-6494.

[22] LI P, WEI Z, WU T, et al. Au-ZnO hybrid nanopyramids and their photocatalytic properties. J. Am. Chem. Soc., 2011, 133(15): 56605663.

[23] BHATTACHARJEe U, MEN L, ROSAlES B A, et al. Using ATTO dyes to probe the photocatalytic activity of Au-CdS nanoparticles. J. Phys. Chem. C, 2017, 121(1): 676-683.

[24] CAO S W, YIN Z, BARBER J, et al. Preparation of Au-BiVO heterogeneous nanostructures as highly efficient visible-light photocatalysts. ACS Appl. Mater. Interfaces, 2012, 4(1): 418-423.

[25] SHANG M, HOU H, GAO F, et al. Mesoporous Ag@ $\mathrm{TiO}_{2}$ nanofibers and their photocatalytic activity for hydrogen evolution. $R S C$
Adv., 2017, 7(48): 30051-30059.

[26] WANG D J, XUE G L, ZHEN Y Z, et al. Monodispersed Ag nanoparticles loaded on the surface of spherical $\mathrm{Bi}_{2} \mathrm{WO}_{6}$ nanoarchitectures with enhanced photocatalytic activities. J. Mater. Chem., 2012, 22(11): 4751-4758.

[27] QAMAR M, ELSAYED R B, ALHOOSHANI K R, et al. Highly efficient and selective oxidation of aromatic alcohols photocatalyzed by nanoporous hierarchical $\mathrm{Pt} / \mathrm{Bi}_{2} \mathrm{WO}_{6}$ in organic solventfree environment. ACS Appl. Mater. Interfaces, 2015, 7(2): 12571269.

[28] ISMAIL A A, BAHNEMANN D W. Mesostructured Pt/TiO nanocomposites as highly active photocatalysts for the photooxidation of dichloroacetic acid. J. Phys. Chem. C, 2011, 115(13): 5784 5791.

[29] XIAO F X. Metal-cluster-decorated $\mathrm{TiO}_{2}$ nanotube arrays: a composite heterostructure toward versatile photocatalytic and photoelectrochemical applications. Small, 2015, 11(S): 554-567.

[30] CHEN Y S, KAMAT P V. Glutathione-capped gold nanoclusters as photosensitizers. visible light-induced hydrogen generation in neutral water. J. Am. Chem. Soc., 2014, 136(16): 6075-6082.

[31] SUBRAMANIAN V, WOLF E E, KAMAT P V. Catalysis with $\mathrm{TiO}_{2}$ /gold nanocomposites. effect of metal particle size on the fermi level equilibration. J. Am. Chem. Soc., 2004, 126(15): 49434950.

[32] WANG Z H, ZHAO S P, ZHU S Y, et al. Photocatalytic synthesis of $\mathrm{M} / \mathrm{Cu}_{2} \mathrm{O}(\mathrm{M}=\mathrm{Ag}, \mathrm{Au})$ heterogeneous nanocrystals and their photocatalytic properties. CrystEngComm., 2011, 13(7): 22622267. 